\title{
Inkjet-printed microtiter plates for portable electrochemical immunoassays
}

\author{
Milica Jović, Yingdi Zhu, Andreas Lesch, Alexandra Bondarenko, Fernando Cortés-Salazar, \\ Frédéric Gumy, Hubert H. Girault *
}

Laboratoire d'Electrochimie Physique et Analytique, École Polytechnique Fédérale de Lausanne, EPFL Valais Wallis, CH-1951 Sion, Switzerland

\section{A R T I C L E I N F O}

\section{Article history:}

Received 9 November 2016

Received in revised form 28 December 2016

Accepted 31 December 2016

Available online 04 January 2017

\section{Keywords:}

Atrazine

Electrochemical detection

Immunoassay

Inkjet-printed

TSH

\begin{abstract}
A B S T R A C T
Herein, we present the large-scale fabrication of multiplexed three-electrode sensors used in a point-of-care device platform that couples a magnetic bead-based immunoassay strategy with amperometric detection for rapid and highly sensitive analysis. The multiplexed sensors consisted of eight independent electrochemical cells, each with a carbon nanotube (CNT) working electrode, CNT counter electrode and a silver-silver chloride quasi-reference electrode. The microchips were fabricated on flexible polyethylene terephthalate (PET) sheets by sequential multilayer inkjet printing (IJP) of silver, CNT and insulator inks that were either simultaneously or subsequently post-processed (e.g. through UV photo-polymerization or photonic curing). Finally, plastic wells were mounted on top of the inkjet-printed patterns to obtain an eight-well microtiter plate where each well had a solution capacity of $50 \mu \mathrm{L}$. Due to the high precision of the IJP process, the microtiter plates showed high reproducibility among the individual electrochemical cells (1-2\% of deviation). Furthermore, the microchips can be reusable for at least up to 20 times as demonstrated herein. In a customized multichannel potentiostat with eight implemented magnets matching the positions of the working electrodes, the electrochemical readout of magnetic bead based sandwich and competitive immunoassays was successfully realized for the detection of thyroid-stimulating hormone (TSH) and atrazine (ATR) in aqueous and urine samples, respectively. The achieved limits of detection for ATR (i.e. $0.01 \mu \mathrm{g} / \mathrm{L}$ ) and TSH (i.e. $0.5 \mu \mathrm{IU} / \mathrm{mL}$ ) demonstrated the potential of the IJP microtiter plates for the environmental and biological quantification of analytes in a very reliable high throughput platform. This work shows that IJP has certainly reached the status of a batch production tool for electroanalytical sensing platforms. (C) 2017 Elsevier B.V. All rights reserved.
\end{abstract}

\section{Introduction}

The development of reliable and cost-effective point-of-care (POC) platforms is important for improving the analytical sensing capabilities, especially in developing countries where it often lacks modern, wellequipped laboratories and fully automated instruments for qualitative and quantitative diagnostics for health care as well as environmental sensing [1]. For instance, rapid and easy-to-use POC devices could help to improve and extend the facilities of the local physicians to diagnose more easily and reliably the patient's diseases and possibly to decide

Abbreviations: Ab, antibody; ALP, alkaline phosphatase; ATR, atrazine; ATR-HRP, atrazine conjugated with horseradish peroxidase; $\mathrm{CE}$, counter electrode; CNT, carbon nanotubes; CV, cyclic voltammetry; ELISA, enzyme-linked immunosorbent assay; HDPE, high-density polyethylene; HRP, horseradish peroxidase; IJP, inkjet printing; MBs, magnetic beads; NP, nanoparticle; PAP, $p$-aminophenol; PAPP, $p$-aminophenol phosphate; PET, polyethylene terephthalate; POC, point-of-care; QRE, quasi-reference electrode; RT, room temperature; TMB, 3,3',5,5'-tetramethylbenzidine; TMB DI, 3,3',5,5'tetramethylbenzidine diimine; TSH, thyroid stimulating hormone; WE, working electrode.

* Corresponding author.

E-mail address: hubert.girault@epfl.ch (H.H. Girault). upon such rapid test to transfer the patient to one of the more advanced, but also expensive laboratories or hospitals [1,2].

Important analytes for POC devices in the third world countries include medical targets (infectious, sexually transmitted and cardiac diseases, cancer) or emerging organic pollutants (pharmaceutical products and pesticides). For instance, the thyroid-stimulating hormone (TSH) is a glycoprotein stimulating thyroidal thyroxin and triiodothyronine in the human organism [3] and can be a marker for different diseases, e.g. subclinical thyroid disease [4] or iodine deficiency disorders [5]. Furthermore, rapid environmental sensing of pesticides and toxins in drinking water is of high importance for a broad range of the population of developing countries being often fixed to only one source of water. For example, atrazine (ATR) is a micropollutant of both ground and surface water, which was widely used in agriculture as an herbicide due to its high effectiveness and low cost [6]. The utilization of ATR was forbidden in Europe in 2003 due to its possible toxic effects [7-10], however ATR is still used in about 60 countries around in Africa, North and South America, Asia and the Middle East. Therefore, significant amounts can be still detected, as classical wastewater treatment plants do not eliminate efficiently ATR, because advanced oxidation processes would be required [8-12]. Despite the fact that recent 
studies presented the evolution of ATR-degrading capabilities in the environment [13], the maximum residue level of ATR in water defined by the WHO (i.e. $0.1 \mu \mathrm{g} / \mathrm{L}$ ) requires sensitive analytical tools such as immunoassay strategies for its fast and reliable detection [14-16]. The enzyme-linked immunosorbent assay (ELISA) relies on the inherent ability of an antibody (Ab) to bind to the specific structure of a molecule, most commonly in form of sandwich or competitive assays. A specific enzyme employed in the assay initiates an enzymatic reaction, whose products can be detected by using various techniques including colorimetric, electrochemical, fluorescence, chemiluminescence [17], surface-plasmon resonance, surface-enhanced Raman scattering [18] or quartz crystal microbalance detection methods [19]. In the electrochemical approach, a current is recorded as a result of an electron transfer from the molecules generated by the enzymatic reaction to a biased electrode. Such approach has attracted considerable interest because of simple instrumentation, easy signal quantification, portability, low cost and miniaturization possibilities [20-23]. For instance, in the typical TSH sandwich assay (Fig. 1a) an Ab against TSH is bound to a support (herein magnetic beads, MBs) and another type of Ab is labelled with the enzyme alkaline phosphatase (here Ab-ALP). When the analyzed sample contains TSH, it will form the immunocomplex with both Abs and the amount of ALP enzyme bound to MBs will increase with increasing TSH concentration. Furthermore, ALP reacts with the $p$ aminophenol phosphate (PAPP) substrate and produces $p$ aminophenol (PAP) that is detected amperometrically. On the other hand, the ATR immunoassay was designed as the competitive immunoassay (Fig. 1b) where the Ab against ATR was bound to the MBs support. ATR present in the sample competes with an ATR-HRP conjugate (atrazine labelled with horseradish peroxidase), which is added to the sample as well, for binding with the Ab. When the analyzed sample contains no ATR, the amount of ATR-HRP bound to MBs will reach a maximum, whereas it will decrease with increasing concentration of the analyte
ATR. ATR-HRP reacts with the 3,3',5,5'-tetramethylbenzidine substrate (TMB) and produces 3,3',5,5'-tetramethylbenzidine diimine (TMB DI), which is electrochemically detected. Since it is a competitive assay, the amperometric signal is inversely proportional to the ATR concentration.

State-of-the-art electrodes in electroanalytical platforms are mainly fabricated by photolithographic and screen-printing techniques which both rely on the usage of mask for each deposited layer. Furthermore, photolitography requires expensive clean room facilities while screen printing suffers from the fact that printing and curing cannot be combined. Recently, inkjet printing has become an attractive alternative for microfabrication. It is an additive manufacturing technique that enables the mask- and contact-less deposition of a variety of functional materials, such as metal and metal oxide nanoparticles, organometallic compounds, conductive polymers, carbonaceous materials, protein microarrays and living cells with high accuracy and micrometer resolution using picoliter droplets [24-35]. The advantages of IJP include low ink waste, easily changeable layouts and the possibility of multi material deposition to create material gradients and multi-layer devices [3638]. Moreover, IJP can reduce the overall fabrication time and costs of an entire device due to the possibility of rapid iterative design changes during the sensor development stage and due to the up-scalability of the production rate from the prototype to the industrial level. In this way, IJP has been used for the fabrication of a broad range of chemical sensors based on gold on paper [39-41] as well as polymer substrates $[27,42,43]$, silver $[30,44]$ polyaniline $[45,46]$ or CNTs $[47-50]$. CNTs are attractive functional materials for sensing platforms due to several advantageous CNT properties, such as good electrical conductivity, chemical and mechanical stability, high flexibility, electrocatalytic activity and reduced surface fouling characteristics for specific applications [51-54]. Recently, we introduced the large-scale production of inkjetprinted CNT electrodes and their application as amperometric sensors

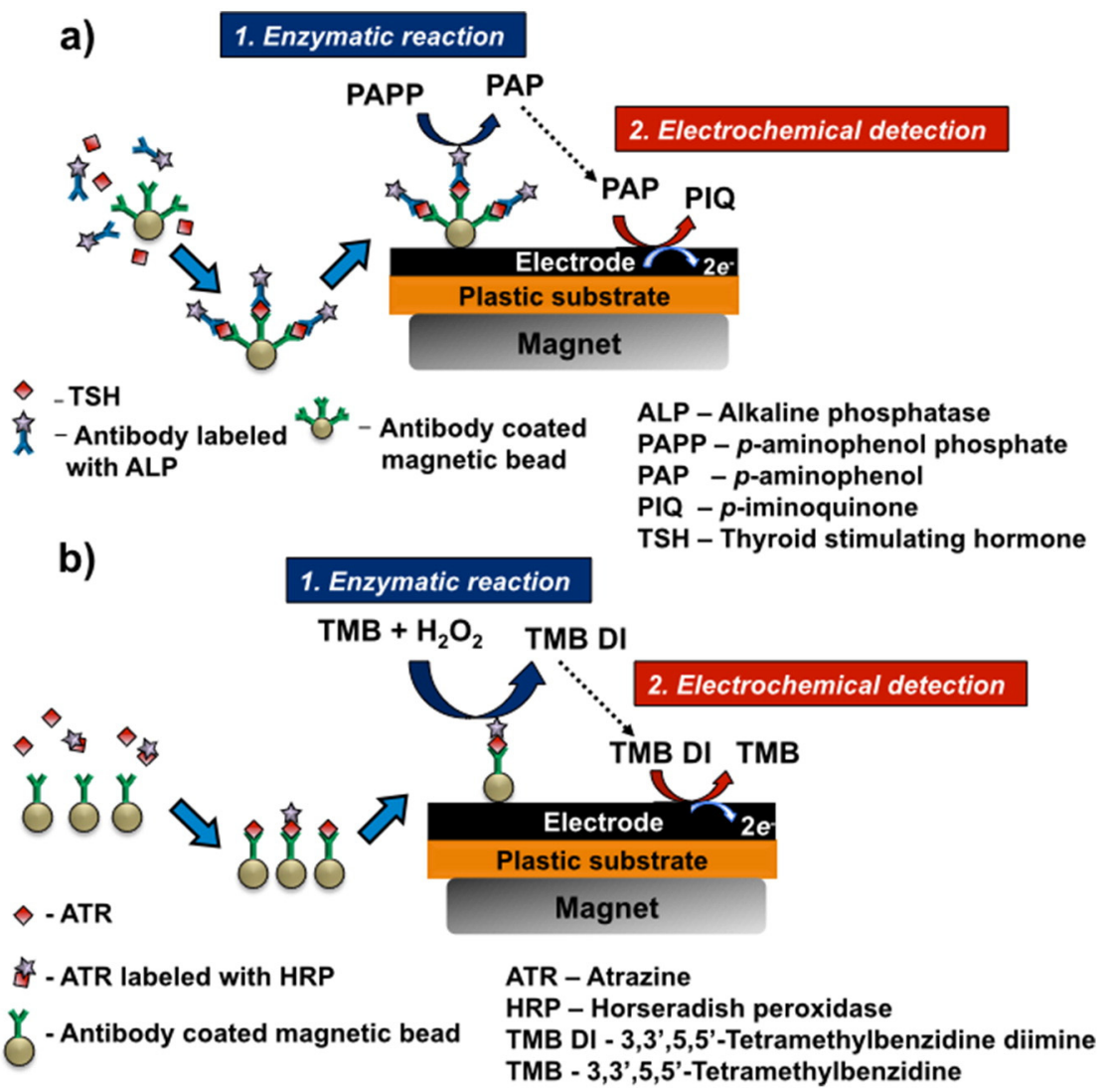

Fig. 1. Schemes of the TSH sandwich immunoassay (a) and ATR competitive immunoassay (b) coupled with electrochemical detection. 
for the detection of antioxidants in blood bags and beverages with matrix independent sensing capabilities after functionalization with an inkjet-printed hydrogel $[29,31,55]$.

Herein, we report inkjet-printed multiplexed three-electrode microchips based on CNT working electrodes, which act as microtiter plates in a point-of-care platform by combining magnetic-bead based immunoassay strategies with electrochemical detection. The large-scale fabrication of the multiplexed electrochemical chips (i.e. eight independent three-electrode cells with CNT working electrode, $\mathrm{Ag} / \mathrm{AgCl}$ quasireference electrode QRE and CNT counter electrode) was comprised of the sequential multilayer deposition from different printheads combined with post-processing steps. The multiplexed sensors were applied for monitoring TSH in human urine and ATR in aqueous samples, using optimized magnetic bead immunoassay protocols based on sandwich and competitive strategies, respectively. Moreover, the IJP microtiter plates showed a high reproducibility and stability that allowed multiple usages of the electrochemical cells of up to 20 times with proper and gentle washing (with DI water), drying (under a stream of nitrogen) and storage procedures (to avoid oxidation of Ag and contamination of CNTs from the environment). The response variability of the microchips was finally $<2 \%$.

\section{Experimental part}

\subsection{Materials}

Jettable nano silver Silverjet DGP-40LT-15C (w/w 30-35\%, Sigma Aldrich, Buchs, Switzerland), CNT dispersion CNTRENE ${ }^{\circledR} 3024$ A3-R (Brewer Science, Rolla, USA) and jettable insulator EMD6201 (Sun Chemical, Carlstadt, USA) were used as inks for IJP. Sodium hypochlorite solution (ACROS Organics, Renningen, Germany, available chlorine 5\%), goat anti-rabbit Ab conjugated with HRP (Ab-HRP) (Invitrogen, Life Technologies, Zug, Switzerland), and rabbit anti-Bovine Ab conjugated with ALP (Ab-ALP) (Sigma-Aldrich, Buchs, Switzerland) were used as received. Deionized water was produced by Milli-Q plus 186 model (Millipore, Zug, Switzerland). The ELISA kit for TSH detection (Immunometrics, London, UK) consisted of standard TSH solutions (purified human TSH lyophilized in horse serum, reconstituted by the addition of deionized water to obtain $60 \mu \mathrm{IU} / \mathrm{mL}$ TSH), washing buffer solution (0.1 M TRIS/ $\mathrm{HCl}$ buffer with $0.5 \%$ sodium azide, $\mathrm{pH} 7.5$ ), MBs coated with $\mathrm{Ab}$ against TSH (MBs-Ab, suspension in $0.05 \mathrm{M}$ phosphate buffer with $0.1 \%$ rat IgG and $0.5 \%$ sodium azide, $\mathrm{pH} 7.4$ ) and a solution with Ab against TSH conjugated with ALP (Ab-ALP, provided as a 55 times concentrate and diluted with $0.05 \mathrm{M}$ phosphate buffer with $0.1 \%$ rat IgG and $0.5 \%$ sodium azide, pH 7.4). 4-aminophenyl phosphate (24 mM PAPP, pH 7.6) substrate solution (DiagnoSwiss, Monthey, Switzerland) was used for electrochemical characterization measurements. Urine samples were kindly provided from the company DiagnoSwiss (Monthey, Switzerland). Samples were stored frozen at $-20^{\circ} \mathrm{C}$. Before the analysis they were exposed to the room temperature (to unfreeze) and then incubated following the protocol from the TSH kit provider. When handling the samples authors always used disposable gloves, while the work areas were disinfected after conducting the experiments. The ELISA kit for ATR detection (Abraxis, Warminster, USA) consisted of standard ATR solutions (0.1, 1.0 and $5.0 \mathrm{ppb}$ in distilled water with preservatives and stabilizers), diluent solution (distilled water with preservative and stabilizers without detectable ATR), washing solution (preserved deionized water), substrate solution (mixture of TMB and $\mathrm{H}_{2} \mathrm{O}_{2}$ in an organic base, $\mathrm{pH}$ 6; concentrations not specified by the provider), MBs coated with Ab against ATR (MBs-Ab, rabbit anti-ATR Ab covalently bound to paramagnetic particles suspended in a buffer solution with preservative and stabilizers, pH 7.5) and ATR-HRP conjugate solution (horseradish peroxidase labelled ATR analog diluted in a buffer solution with preservative and stabilizers, pH 7.5). Ferrocene methanol (97\%) and potassium nitrate were obtained from Sigma Aldrich (Buchs, Switzerland). Water samples were taken from municipal water supply in Sion and used without any treatment.

\subsection{Microtiter plate fabrication}

Electrochemical microtiter plates were fabricated on a $250 \mu \mathrm{m}$-thick PET substrate (Goodfellow, Huntingdon, England) using an X-Serie CeraPrinter (Ceradrop, Limoges, France) comprising permanently three parallel piezoelectric drop-on-demand printheads, i.e., two QClass Sapphire QS-256 each with 256 individually addressable nozzles for either 10,30 or $80 \mathrm{pL}$ nominal droplet volumes and one disposable DMC-11610 cartridge containing 16 individually addressable nozzles with $10 \mathrm{pL}$ nominal droplet volume (all print heads from Dimatix Fujifilm, Lebanon, NH, USA). The microchip design was created with the CeraSlice software from Ceradrop. Custom waveforms were developed for all inks to gain optimal droplet formation. Several print parameters were optimized including the horizontal drop distance on the substrate, substrate temperature and printhead height. In brief, the substrate temperature was increased to $60^{\circ} \mathrm{C}$ for the IJP of both $\mathrm{Ag}$ and CNT inks to accelerate ink solvent evaporation and ensure highly resolved patterns. The silver ink was printed with a Q-Class Sapphire QS-256 printhead, dried and cured "in-line" in the CeraPrinter post-processing chamber using a high intensity light pulse from a xenon flash lamp provided by the PulseForge 1300 photonic curing station (NovaCentrix, Austin, TX, USA). The CNT ink and UV curable dielectric ink were printed using disposable cartridges. The UV curable dielectric ink was simultaneously printed and photo-polymerized with a UV LED lamp (FireEdge FE300 380-420 nm; Phoseon Technology) integrated into the according printhead slot of the CeraPrinter. After printing and post-processing, the bare CNT electrodes were ready-to-use for the optical as well as electrochemical characterizations and immunoassay-based detection. The optical characterization of the fabricated microchips was performed by using the fabrication analyzer camera inside the CeraPrinter and a Keyence VK 8700 laser scanning microscope (Keyence, Osaka, Japan). The plastic pieces used to obtain the wells on the microtiter plates were produced from high-density polyethylene (HDPE) sheets of (dimensions $77 \mathrm{~mm} \times 15 \mathrm{~mm} \times 3 \mathrm{~mm}$ ) by drilling mechanically well-defined holes. Afterwards, the plastic pieces with eight wells were glued directly on the PET foil with printed electrodes using a double side adhesive tape (DigiKey 3M9726-ND) with pre-prepared holes of the same diameter as the wells. The tape was mechanically and chemically stable under the applied experimental, sample and microchip preparations in order to avoid any leakage of sample solution.

\subsection{Electrochemical characterization of IJP microtiter plates}

The IJP microchips were electrochemically characterized by using cyclic voltammetry (CV) in FcMeOH solution (2 mM, $\left.0.1 \mathrm{M} \mathrm{KNO}_{3}\right)$, as well as in PAPP (24 mM, pH 7.6) and TMB ( $\mathrm{pH} 6$, concentration not specified by provider) commercial substrate solutions in the presence and absence of MBs. For the measurements with the MBs, $50 \mu \mathrm{L}$ of each MBs suspension (from the TSH and ATR kits, respectively) was transferred into a vial, sedimented and separated from the supernatant using a magnetic separation stand (Promega AG Dübendorf, Switzerland). Subsequently, the MBs were resuspended in $50 \mu \mathrm{L}$ of PAPP or TMB commercial stock solution and transferred into the microchip wells. Substrate solutions without MBs were used as blank samples. CV experiments were carried out using a customized housing with embedded OEM EmStat MUX8 potentiostat (PalmSens, Houten, Netherlands). The microchip holder contained integrated magnets located directly below the working electrodes. In this way, the magnetic beads with the immunoassay complex were concentrated close to the CNT electrode surface leading to an amplification of the electrochemical signal.

Moreover, the signal reproducibility was determined by the amperometric detection of PAP and TMB DI. For this purpose, $1 \mathrm{~mL}$ of PAPP was incubated with $0.5 \mu \mathrm{L}$ of Ab-ALP stock solution and $50 \mu \mathrm{L}$ of TMB $+\mathrm{H}_{2} \mathrm{O}_{2}$ was incubated with $25 \mu$ L ATR-HRP overnight to complete the enzymatic production of PAP and TMB DI, respectively. 


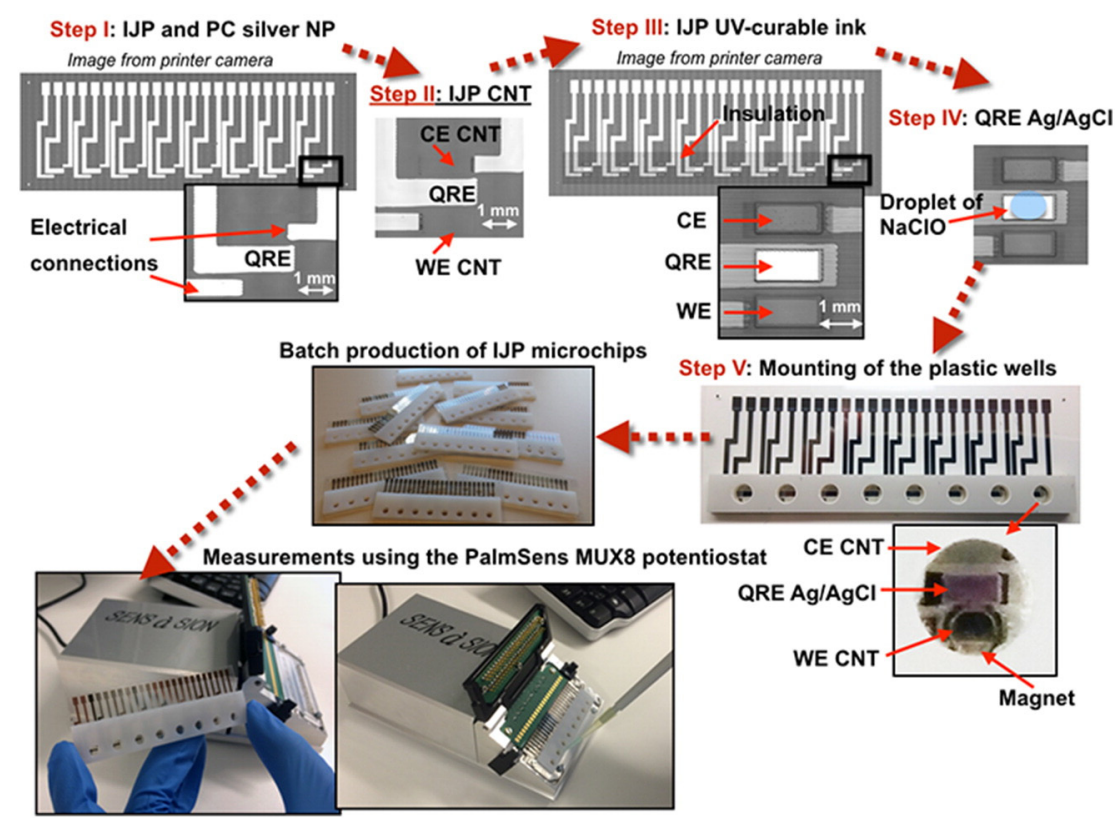

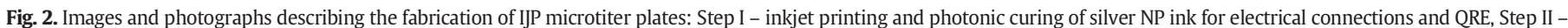

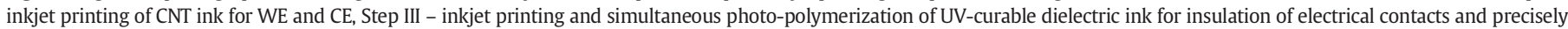

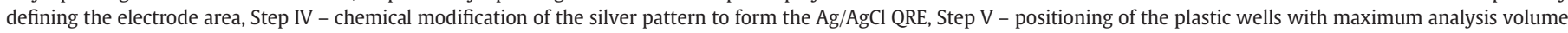

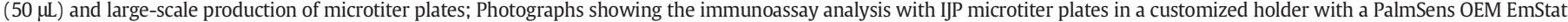
MUX8 multichannel potentiostat and implemented magnets matching the positions of the WE.

Thereafter, $40 \mu \mathrm{L}$ of the obtained solution was transferred into each well of the microchip and the electrochemical signal was read out simultaneously in all 8 wells at the corresponding optimal electrode potential after $60 \mathrm{~s}$.

\subsection{TSH and ATR quantification in IJP microtiter plates}

Calibration curves for TSH were obtained for five different concentrations $(0,3.75,7.5,15$ and $30 \mu \mathrm{IU} / \mathrm{mL}$ TSH) in triplicate (each replicate in different microtiter well) following the commercial protocol. Briefly, $10 \mu \mathrm{L}$ of standard TSH solution and $10 \mu \mathrm{L}$ of MBs-Ab suspension were added into the vials and incubated during $15 \mathrm{~min}$ at $37{ }^{\circ} \mathrm{C}$ under stirring (1000 rpm). The immunocomplex was sedimented and the supernatant discarded. Then $10 \mu \mathrm{L}$ of Ab-ALP solution was added and incubated for $1 \mathrm{~h}$ at $37{ }^{\circ} \mathrm{C}$ under stirring (1000 rpm). The system was then rinsed 3 times with $30 \mu \mathrm{L}$ of the washing solution. Finally, $50 \mu \mathrm{L}$ of the PAPP substrate solution was added into each vial, the suspension was transferred into the microchip well and the CNT electrode was biased at $0.05 \mathrm{~V}$. The calibration curve was also performed in urine samples (without detectable TSH). Thereafter, the recovery test was carried out using urine spiked with $1 \mu \mathrm{IU} / \mathrm{mL}, 5 \mu \mathrm{IU} / \mathrm{mL}$ and $10 \mu \mathrm{IU} / \mathrm{mL}$ of TSH and analyzed in triplicate using one fresh microtiter well for each measurement.

ATR calibration curves were obtained for five different concentrations $(0,0.05,0.2,0.5$ and $1 \mathrm{ppb}$ ATR $)$ in triplicate following an optimized protocol (SI-I). Briefly, $250 \mu \mathrm{L}$ of standard ATR solution, $250 \mu \mathrm{L}$ of ATR-HRP and $250 \mu \mathrm{L}$ of MBs-Ab suspension were added into the vials and incubated for $15 \mathrm{~min}$ at $37^{\circ} \mathrm{C}$ under stirring ( $1000 \mathrm{rpm}$ ). Thereafter, the system was rinsed with $1000 \mu \mathrm{L}, 500 \mu \mathrm{L}$ and $100 \mu \mathrm{L}$ of the washing solution. Finally, $50 \mu \mathrm{L}$ of the TMB substrate solution was added into each vial, the suspension was transferred into the microchip and the CNT electrode was biased at $-0.1 \mathrm{~V}$. The water samples from Sion municipal supply spiked with $0.05 \mu \mathrm{g} / \mathrm{L}$ and $0.1 \mu \mathrm{g} / \mathrm{L}$ of ATR were analyzed in triplicate using one fresh microtiter well for each measurement.
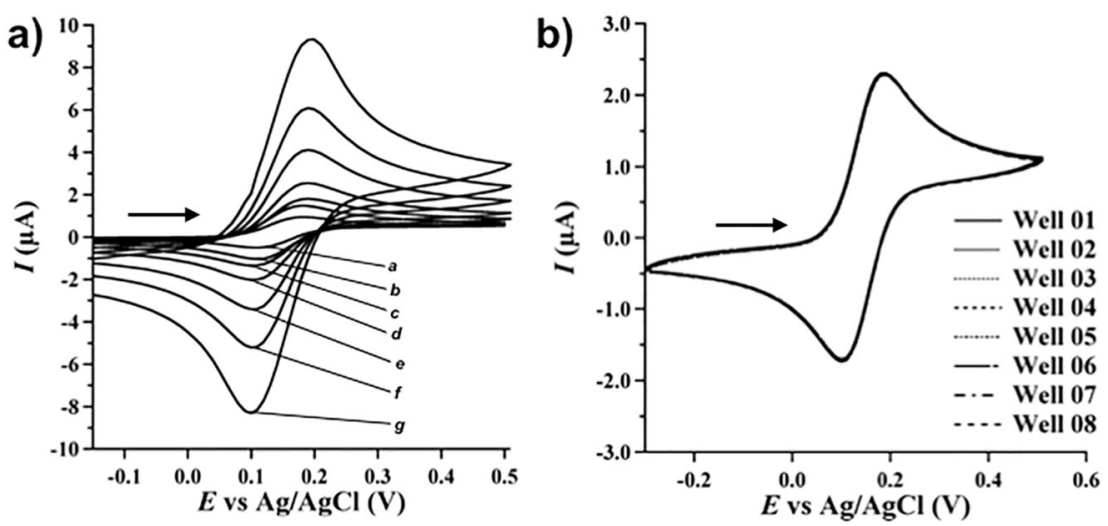

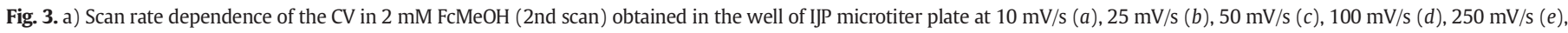

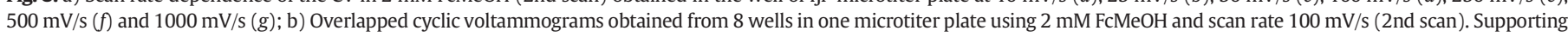
electrolyte $0.1 \mathrm{M} \mathrm{KNO}_{3}$. 

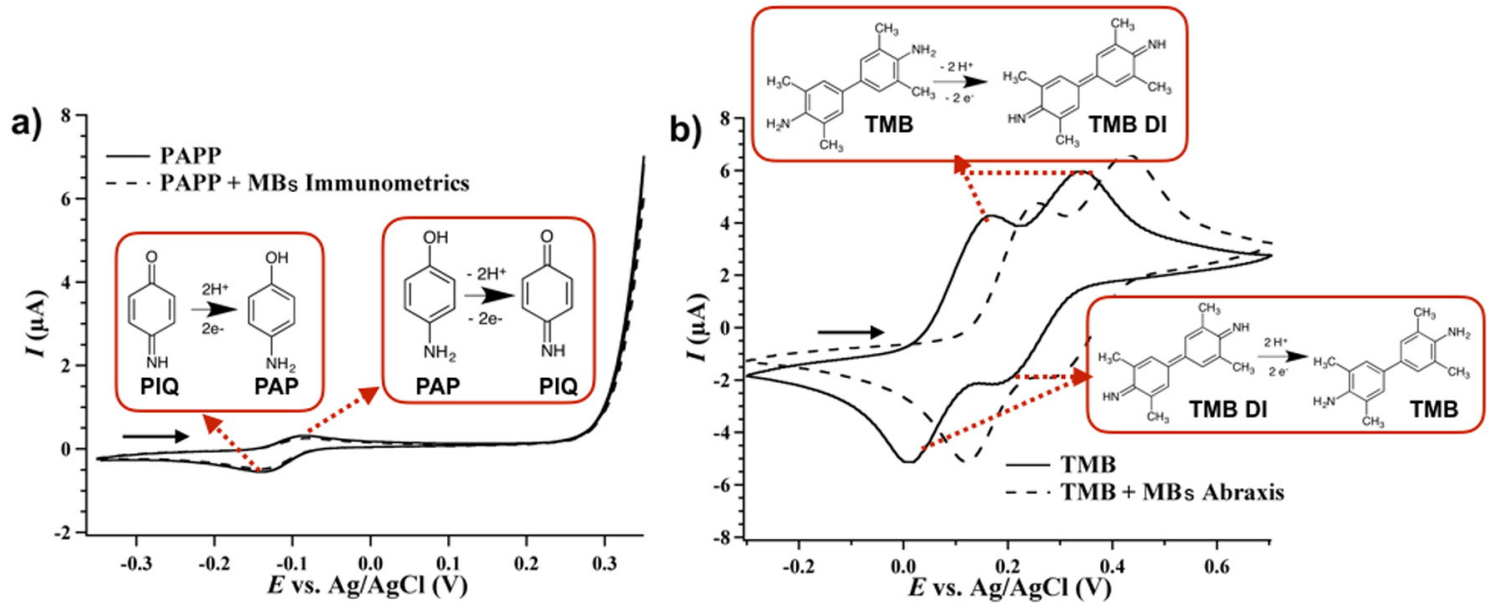

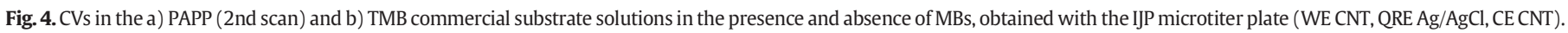
Scan rate was $50 \mathrm{mV} / \mathrm{s}$.

\section{Results and discussion}

\subsection{Fabrication and characterization of IJP microtiter plates}

The outcome of the subsequent inkjet printing steps for the production of the microtiter plate is shown in Fig. 2. The first step was printing of the silver layer for the electrical connections and quasi-reference electrode (Step I). The silver nanoparticle (NP) ink was converted into conductive and well-adhered traces within fractions of a second by the photonic flash sintering. The principle of this technique is the selective heating of the Ag nanoparticles by the absorption of the emitted light for which the substrate is transparent $[56,57]$. Just after, CNT patterns that only slightly overlapped with Ag for the electrical connection were printed forming the CNT working and counter electrodes (Step II). The UV curable dielectric ink was printed for the insulation of the electrical Ag circuit and to define precisely all three active geometric electrode areas, which were equally $A_{\mathrm{g}} \approx 0.72 \mathrm{~mm}^{2}$ (Step III). Silver itself is not a suitable material for a stable and reliable reference electrode (RE) due to the rapid chemical modification of the Ag surface (e.g., by oxidation) causing a drift of the potential during measurements. In the past few years, many efforts were made to develop and miniaturize reliable REs for sensors and lab-on-a-chip devices. Recently, a new class of SSRE without solid junction has been fabricated by inkjet printing of silver followed by either electrochemical or chemical formation of an $\mathrm{AgCl}$ layer [39,44]. Following the protocol from da Silva et al. [44], $1 \mu \mathrm{L}$ of bleach solution was drop casted onto the as printed and cured Ag patterns where $\mathrm{AgCl}$ was formed within 3 min (Step IV) [55]. The change in the color from shiny silver to dark purple indicated clearly the transformation of $\mathrm{Ag}$ into $\mathrm{AgCl}$. Afterwards, the electrodes were washed with deionized water and dried with a stream of nitrogen. The obtained $\mathrm{Ag} /$ $\mathrm{AgCl}$ QREs were ready-to-use and showed long-term stability during dry storage of $>3$ months with $\Delta E<0.01 \mathrm{~V}$ against a commercial $\mathrm{Ag}$ / $\mathrm{AgCl}(3 \mathrm{M} \mathrm{KCl}) \mathrm{RE}$. The stability of the QRE is extremely important and certainly influenced by the chloride activity in solution. It should be noted that all sample solutions analyzed herein (both for TSH and ATR assays) were prepared reproducibly so that the concentration of chloride anions or other anions, which could have an influence on the potential of the $\mathrm{Ag} / \mathrm{AgCl} \mathrm{QRE}$, were identical or very similar. Indeed, a shift of the peak potentials for the used redox active species between measurements was not observed. The fabrication process was finished by placing a plastic well chip to obtain microtiter plates composed of eight independent electrochemical cells with a maximum filling volume of $50 \mu \mathrm{L}$ (Step V). Please note that the electrode areas were only defined by the highly reproducible inkjet printing process, while the wells, in diameter slightly larger than the active electrode areas of the microchip, defined the sample volume.

In order to characterise the general electrochemical response of the IJP microtiter plates, CVs were recorded using $\mathrm{FcMeOH}$ as a redox mediator in a $2 \mathrm{mM}$ solution $\left(0.1 \mathrm{M} \mathrm{KNO}_{3}\right)$. The CVs showed the one electron oxidation of $\mathrm{FcMeOH}$ to $\mathrm{FcMeOH}^{+}$in the positive forward scan and the reduction of $\mathrm{FcMeOH}^{+}$to $\mathrm{FcMeOH}$ in the reverse negative scan (Fig. 3a). CVs recorded with different scan rates from 10 to $1000 \mathrm{mV} / \mathrm{s}$ indicated a quasi-reversible electrochemical behaviour with small deviations from theory. As discussed previously, such behaviour could be explained by an IR drop along the CNT electrode, kinetic limitations and non-linear diffusion effects due to microscopic inhomogeneities [29]. The later could be seen by the peak potential separation which exceeds the theoretical value of $59 \mathrm{mV}$ for a fast one-electron transfer by $\sim 15 \mathrm{mV}$.
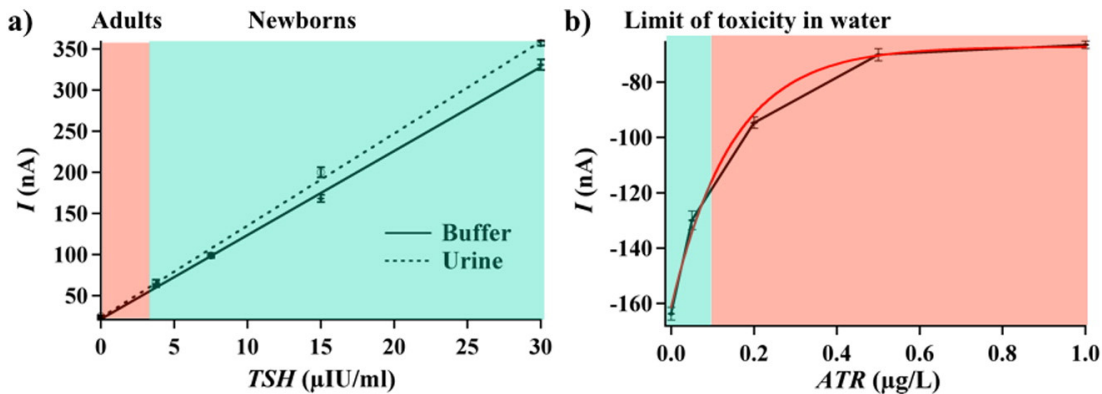

Fig. 5. Calibration curves for the amperometric detection of TSH in buffer and urine a), and ATR in water b), using the immunoassay protocols combined with IJP microchips. 
Table 1

Results of the recovery test for TSH and ATR in real samples analyzed by the immunoassay coupled with amperometric detection in the inkjet-printed microchips.

\begin{tabular}{|c|c|c|c|}
\hline Analyzed sample & Compound & Spiked concentration & Detected concentration \\
\hline Urine sample & TSH & $10 \mu \mathrm{IU} / \mathrm{mL}$ & $9.98 \pm 0.14 \mu \mathrm{IU} / \mathrm{mL}$ \\
\hline Urine sample & TSH & $1 \mu \mathrm{IU} / \mathrm{mL}$ & $1.10 \pm 0.04 \mu \mathrm{IU} / \mathrm{mL}$ \\
\hline Urine sample & TSH & $0 \mu \mathrm{IU} / \mathrm{mL}$ & Not detected \\
\hline Tap water & ATR & $0.1 \mu \mathrm{g} / \mathrm{L}$ & $0.099 \pm 0.001 \mu \mathrm{g} / \mathrm{L}$ \\
\hline Tap water & ATR & $0.05 \mu \mathrm{g} / \mathrm{L}$ & $0.051 \pm 0.001 \mu \mathrm{g} / \mathrm{L}$ \\
\hline Tap water & ATR & $0 \mu \mathrm{g} / \mathrm{L}$ & Not detected \\
\hline
\end{tabular}

Additionally, the CV curves recorded in eight different wells in one microtiter plate with a scan rate of $100 \mathrm{mV} / \mathrm{s}$ (Fig. 3b) overlap with very high reproducibility thanks to the well-defined CNT electrode area.

The microchips were then characterized for the immunoassay application and Fig. 4a shows typical second cycles of CVs recorded in the PAPP sample solution after the electrochemical generation of initially absent PAP from PAPP. Briefly, PAPP is irreversibly oxidised at the electrode to para-iminoquinone (PIQ) at around $0.4 \mathrm{~V}$, which is electrochemically reduced to PAP at $-0.15 \mathrm{~V}$. In the second CV scan, PAP is electrochemically oxidised at around $-0.1 \mathrm{~V}$. The addition of MBs to the PAPP solution did not present any influence on the CVs and the PAP detection can be performed at the potential equal to $0.05 \mathrm{~V}$. The CVs for the TMB substrate solution (Fig. $4 \mathrm{~b}$ ) presented two oxidation peaks corresponding to the two-electron transfer reaction of TMB [58]. When using the IJP microchip in the absence of any MBs, the TMB oxidation peaks can be observed at potentials around $0.15 \mathrm{~V}$ and $0.35 \mathrm{~V}$ while the TMB DI reduction takes place at around $0.2 \mathrm{~V}$ and $0 \mathrm{~V}$. By adding MBs into the system the measured redox potentials of TMB were shifted by approximately $0.1 \mathrm{~V}$ to higher values, probably due to poor polymeric coating of $\mathrm{Fe}_{3} \mathrm{O}_{4}$ core in MBs (for more details see SI-II). However, the amperometric detection of enzymatically produced TMB DI (i.e. the analytical signal of the ATR immunoassay) could be performed at $-0.1 \mathrm{~V}$.

Afterwards, the IJP microtiter plates were characterized in terms of the reproducibility of the amperometric signal by detecting PAP and TMB DI formed in the enzymatic reaction of PAPP with ALP and TMB with ATR-HRP, respectively (Fig. S4, experimental details in Section 2.3). The recorded current for each well after $60 \mathrm{~s}$ of applying the according working potentials was plotted. The standard deviations of the detected current from 8 wells varied only by $1.8 \%$ (PAP) and $2.1 \%$ (TMB) demonstrating that the microchips can be further implemented for the parallel immunoassays analysis. The original measurement plots can be found in Section SI-III (Fig. S3).

\subsection{Quantification of TSH and ATR in IJP microtiter plates}

The calibration curves for TSH detection in buffer and urine samples were obtained measuring five different concentrations in triplicate (Fig. 5a) and identifying the optimized potentials for the amperometric detection as reported vide supra. The calibration curve with urine samples did not show significant matrix interference on the sensitivity of the assay. Therefore, the IJP microchips can be used reliably for the analysis of a broad range of real biological samples. The limit of detection of the assay was equal to the one announced for the commercial kit, i.e. $0.5 \mu \mathrm{IU} / \mathrm{mL}$. Normal TSH levels in blood according to the American Association of Clinical Endocrinologists (AACE) range from 0.4 to $2.5 \mu \mathrm{IU} / \mathrm{mL}$ for adults, and can be as high as $39 \mu \mathrm{IU} / \mathrm{mL}$ for newborns [59].

The commercial kit for ATR was designed for colorimetric detection and the assay protocol needed to be adjusted and optimized for the electrochemical detection. The calibration curve for ATR detection was performed with five different concentrations in triplicate (Fig. 5b). It was shown that the limit of detection was equal to $0.01 \mu \mathrm{g} / \mathrm{L}$, which is 10 times lower than the limit of toxicity of ATR in water [22].

Immunoassay protocols and calibration curves for TSH and ATR detection were further applied for the analysis of real samples, namely urine and tap water spiked with known amounts of TSH and ATR, respectively. The experimental data showed high recovery percentage of both compounds: 95-110\% (Table 1). Moreover, the IJP microtiter plates showed a high reproducibility and stability that allows multiple usage of the electrochemical cells of up to 20 times with proper washing (with DI water), drying (under a stream of nitrogen) and storage procedures (to avoid oxidation of $\mathrm{Ag}$ and contamination of CNTs from the environment). The response variability of the microchips was finally $<2 \%$. Long-term stability of IJP plates was evaluated by recording the consecutive immunoassay experiments (SI-IV), while on-going studies are exploring the microtiter shelf-life which is currently $>6$ months. The plates should be kept in plastic bags or boxes, prior or between the usages, in order to prevent electrical connection problems due to the oxidation of silver connection pads and formation of resistive silver oxide or contamination of the CNTs from organic molecules present in traces in the environment. Thus, the IJP microchip proved to be a good amperometric platform that can be integrated to different types of the immunoassay for the TSH and ATR monitoring.

The methodologies for measuring TSH have experienced many improvements over the last four decades with respect to the achieved functional sensitivity limits. The most popular assay had been the radioimmunoassay (e.g. TSH immunoradiometric assay IRMA with LOD $0.06 \mu \mathrm{IU} / \mathrm{mL}$ ), colorimetric assay (LOD $0.05 \mu \mathrm{IU} /$ $\mathrm{mL}$ ), immunofluorimetric assay [60], chemiluminescence/electrochemiluminescence assays [61-64] and recently introduced surfaceenhanced Raman spectroscopy (SERS)-based lateral flow assay (LFA) strips [18]. Regarding the ATR detection, several studies based on electrochemical or fluorescence immunoassays have been reported [6568]. In general, the approach presented in this study has the advantage of being more sensitive compared to radiometric and colorimetric assays. Moreover, the presented system is small and can be made portable compared to the relatively large and expensive chemiluminescent and fluorescent equipment, and it requires small sample volumes that are not influenced by matrix color background or $\mathrm{O}_{2}$ content as fluorescence and chemiluminescence strategies can be.

\section{Conclusions}

In summary, we have demonstrated the large-scale fabrication of microtiter plates using the IJP technology in combination with several in-line post-processing techniques. The microtiter plates consisted of 8 independent electrochemical cells each with one CNT working electrode, $\mathrm{Ag} / \mathrm{AgCl}$ quasi-reference electrode and CNT counter electrode. These microchips presented high reproducibility of the recorded signals between the wells (RSD 1-2\%) and fast kinetics for PAP and TMB detection thanks to the physicochemical properties of the CNTs. The microtiter plates were successfully implemented for the environmental and clinical monitoring platforms as exemplarily shown for the immunoassay-based electrochemical detection of ATR and TSH in water and urine samples, respectively. The presented inkjet printing process demonstrates the big potential of this technology to design, develop and produce electrode chips on an industrial scale with high reproducibility. It can be foreseen, that inkjet printing will offer many new opportunities for the fabrication of electroanalytical sensors thanks to its high flexibility in electrode design, its high accuracy, minimum material usage and automated multilayer processing. Together with affordable measurement units and simpleto-use software, the presented digital production and measurement 
approaches become very attractive, in particular for biological and medical sensor applications in developing countries.

\section{Acknowledgments}

The authors thank Dr. Priscille Giron from DiagnoSwiss, Monthey, Switzerland for kindly providing us the urine samples and kit used for TSH detection. M. J. thanks NanoTera (RTD Project Envirobot). A. L. thanks the Swiss National Science Foundation (SNSF project No. 154297). The technical support from Ceradrop and NovaCentrix is acknowledged.

\section{Appendix A. Supplementary data}

Supplementary data to this article can be found online at http://dx. doi.org/10.1016/j.jelechem.2016.12.051.

\section{References}

[1] R.W. Peeling, D. Mabey, Point-of-care tests for diagnosing infections in the developing world, Clin. Microbiol. Infect. 16 (2010) 1062-1069.

[2] S. Sharma, J. Zapatero-Rodríguez, P. Estrela, R. O'Kennedy, Point-of-care diagnostics in low resource settings: present status and future role of microfluidics, Biosensors 5 (2015) 577-601.

[3] O.P. Soldin, S.H. Chung, C. Colie, The use of TSH in determining thyroid disease: how does it impact the practice of medicine in pregnancy? J. Thyroid Res. 2013 (2013) 148157.

[4] M.I. Surks, E. Ortiz, G.H. Daniels, C.T. Sawin, R.H. Cobin, J.A. Franklyn, J.M. Hershman, K.D. Burman, M.A. Denke, R.S. Cooper, N.J. Weissman, Subclinical thyroid disease: scientific review and guidelines for diagnosis and management, JAMA 291 (2004) 228-238.

[5] F. Delange, The disorders induced by iodine deficiency, Thyroid 4 (1994) 107-128.

[6] R.P. Schwarzenbach, B.I. Escher, K. Fenner, T.B. Hofstetter, C.A. Johnson, U. von Gunten, B. Wehrli, The challenge of micropollutants in aquatic systems, Science 313 (2006).

[7] J.B. Sass, A. Colangelo, European Union bans atrazine, while the United States negotiates continued use, Int. J. Occup. Environ. Health 12 (2013) 260-267.

[8] Y. Luo, W. Guo, H.H. Ngo, L.D. Nghiem, F.I. Hai, J. Zhang, S. Liang, X.C. Wang, A review on the occurrence of micropollutants in the aquatic environment and their fate and removal during wastewater treatment, Sci. Total Environ. (2014) 619-641.

[9] L. Bu, Z. Shi, S. Zhou, Modeling of Fe(II)-activated persulfate oxidation using atrazine as a target contaminant, Sep. Purif. Technol. 169 (2016) 59-65.

[10] S. Komtchou, A. Dirany, P. Drogui, N. Delegan, M.A.E. Khakani, D. Robert, P. Lafrance, Degradation of atrazine in aqueous solution with electrophotocatalytic process using $\mathrm{TiO}_{2}-$ x photoanode, Chemosphere 157 (2016) 79-88.

[11] X. Kong, J. Jiang, J. Ma, Y. Yang, W. Liu, Y. Liu, Degradation of atrazine by UV/chlorine: efficiency, influencing factors and products, Water Res. 90 (2016) 15-23.

[12] S. Zhou, L. Bu, Z. Shi, C. Bi, Q. Yi, A novel advanced oxidation process using iron electrodes and ozone in atrazine degradation: Performance and mechanism, Chem. Eng. J. 306 (2016) 719-725.

[13] N. Udiković-Kolić, C. Scott, F. Martin-Laurent, Evolution of atrazine-degrading capabilities in the environment, Appl. Microbiol. Biotechnol. 96 (2012) 1175-1189.

[14] H.C. Liang, N. Bilon, M.T. Hay, Analytical methods for pesticide residues, Water Environ. Res. 85 (2013) 2114-2138.

[15] M. Tudorache, A. Tencaliec, C. Bala, Magnetic beads-based immunoassay as a sensitive alternative for atrazine analysis, Talanta 77 (2008) 839-843.

[16] Z. Qie, J. Bai, B. Xie, L. Yuan, N. Song, Y. Peng, X. Fan, H. Zhou, F. Chen, S. Li, B. Ning, Z. Gao, Sensitive detection of atrazine in tap water using TELISA, Analyst 140 (2015) 5220-5226.

[17] Q. Xiaoa, H. Lib, J.-M. Linb, Development of a highly sensitive magnetic particle-based chemiluminescence enzyme immunoassay for thyroid stimulating hormone and comparison with two other immunoassays, Clin. Chim. Acta 411 (2010) 1151-1153.

[18] S. Choi, J. Hwang, S. Lee, D.W. Lim, H. Joo, J. Choo, Quantitative analysis of thyroidstimulating hormone (TSH) using SERS-based lateral flow immunoassay, Sens. Actuators B 240 (2017) 358-364.

[19] I.A. Darwish, Immunoassay methods and their applications in pharmaceutical analysis: basic methodology and recent advances, Int. J. Biol. Sci. 2 (2006) 217-235.

[20] B.V. Chikkaveeraiah, A.A. Bhirde, N.Y. Morgan, H.S. Eden, X.Y. Chen, Electrochemica immunosensors for detection of cancer protein biomarkers, ACS Nano 6 (2012) 6546-6561.

[21] N.J. Ronkainen, S.L. Okon, Nanomaterial-based electrochemical immunosensors for clinically significant biomarkers, Materials 7 (2014) 4669-4709.

[22] X. Liu, W.-J. Li, L. Li, Y. Yang, L.-G. Mao, Z. Peng, A label-free electrochemica immunosensor based on goldnanoparticles for direct detection of atrazine, Sens. Actuators B 191 (2014) 408-414.

[23] B.S. Munge, T. Stracensky, K. Gamez, D. DiBiase, J.F. Rusling, Multiplex Immunosensor Arrays for Electrochemical Detection of Cancer Biomarker Proteins, Electroanalysis 2016 http://dx.doi.org/10.1002/elan.201600183.

[24] G. Cummins, M.P.Y. Desmulliez, Inkjet printing of conductive materials: a review, Circuit World 38 (2012) 193-213.
[25] V. Romanov, S.N. Davidoff, A.R. Miles, D.W. Grainger, B.K. Gale, B.D. Brooks, A critical comparison of protein microarray fabrication technologies, Analyst 139 (2014) 1303-1326

[26] A. Dias, D. Kingsley, D. Corr, Recent advances in bioprinting and applications for biosensing, Biosensors 4 (2014) 111-136.

[27] A. Moya, E. Sowade, F.J. del Campo, K.Y. Mitra, E. Ramon, R. Villa, R.R. Baumann, G. Gabriel, All-inkjet-printed dissolved oxygen sensors on flexible plastic substrates, Org. Electron. 39 (2016) 168-176.

[28] K. Rajan, I. Roppolo, A. Chiappone, S. Bocchini, D. Perrone, A. Chiolerio, Silver nanoparticle ink technology: state of the art, Nanotechnol. Sci. Appl. 9 (2016) 1-13.

[29] A. Lesch, F. Cortés-Salazar, M. Prudent, J. Delobel, S. Rastgar, N. Lion, J.-D. Tissot, P. Tacchini, H.H. Girault, Large scale inkjet-printing of carbon nanotubes electrodes for antioxidant assays in blood bags, J. Electroanal. Chem. 717-718 (2014) 61-68.

[30] M. Jović, F. Cortés-Salazar, A. Lesch, V. Amstutz, H. Bi, H.H. Girault, Electrochemical detection of free chlorine at inkjet printed silver electrodes, J. Electroanal. Chem. 756 (2015) 171-178

[31] A. Lesch, F. Cortés-Salazar, V. Amstutz, P. Tacchini, H.H. Girault, Inkjet printed nanohydrogel coated carbon nanotubes electrodes for matrix independent sensing, Anal. Chem. 87 (2015) 1026-1033.

[32] N. Komuro, S. Takaki, K. Suzuki, D. Citterio, Inkjet printed (bio)chemical sensing devices, Anal. Bioanal. Chem. 405 (2013) 5785-5805.

[33] J. Li, F. Rossignol, J. Macdonald, Inkjet printing for biosensor fabrication: combining chemistry and technology for advanced manufacturing, Lab Chip 15 (2015) 2538-2558.

[34] E. Luan, Z. Zheng, X. Li, H. Gu, S. Li, Inkjet-assisted layer-by-layer printing of quantum dot/enzyme microarrays for highly sensitive detection of organophosphorous pesticides, Anal. Chim. Acta 916 (2016) 77-83.

[35] A. Kamyshny, S. Magdassi, Conductive nanomaterials for printed electronics, Small 10 (2014) 3515-3535.

[36] S. Khan, L. Lorenzelli, R.S. Dahiya, Technologies for printing sensors and electronics over large flexible substrates: a review, IEEE Sensors Journal 15 (2015) 3164-3185.

[37] D.K. Kampouris, R.O. Kadara, N. Jenkinson, C.E. Banks, Screen printed electrochemical platforms for pH sensing, Anal. Methods 1 (2009) 25-28.

[38] S. Laschi, I. Palchetti, M. Mascini, Gold-based screen-printed sensor for detection of trace lead, Sens. Actuators B 114 (2006) 460-465.

[39] A. Määttänen, U. Vanamo, P. Ihalainen, P. Pulkkinen, H. Tenhu, J. Bobacka, J. Peltonen, A low-cost paper-based inkjet-printed platform for electrochemical analyses, Sens. Actuators B 177 (2013) 153-162.

[40] P. Sjöberg, A. Määttänen, U. Vanamo, M. Novell, P. Ihalainen, F.J. Andrade, J. Bobacka, J. Peltonen, Paper-based potentiometric ion sensors constructed on ink-jet printed gold electrodes, Sens. Actuators B 224 (2016) 325-332.

[41] M. Borzenkov, A.K. Määttänen, P. Ihalainen, M. Collini, E. Cabrini, G. Dacarro, P. Pallavicini, G. Chirico, Fabrication of inkjet printed gold nanostar patterns with photothermal properties on paper substrate, ACS Appl. Mater. Interfaces 8 (2016) 9909-9916.

[42] G.C. Jensen, C.E. Krause, G. Sotzing, J.F. Rusling, Inkjet-printed gold nanoparticle electrochemical arrays on plastic. Application to immunodetection of a cancer biomarker protein, Phys. Chem. Chem. Phys. 13 (2011) 4888-4894.

[43] A. Määttänen, P. Ihalainen, P. Pulkkinen, S. Wang, H. Tenhu, P. Juoko, Inkjet-printed gold electrodes on paper: characterization and functionalization, ACS Appl. Mater. Interfaces 4 (2012) 955-964.

[44] E.T. da Silva, S. Miserere, L.T. Kubota, A. Merkoci, Simple on-plastic/paper inkjetprinted solid-state $\mathrm{Ag} / \mathrm{AgCl}$ pseudoreference electrode, Anal. Chem. 86 (2014) 10531-10534.

[45] T. Hibbard, K. Crowley, F. Kelly, F.L. Ward, J. Holian, AJ. Watson, A.J. Killard, Point of care monitoring of hemodialysis patients with a breath ammonia measurement device based on printed polyaniline nanoparticle sensors, Anal. Chem. 85 (2013) 12158-12165.

[46] C. Bardpho, P. Rattanarat, W. Siangproh, O. Chailapakul, Ultra-high performance liquid chromatographic determination of antioxidants in teas using inkjet-printed graphene-polyaniline electrode, Talanta 148 (2015) 673-679.

[47] R.P. Tortorich, S. Song, J.-W. Choi, Inkjet-printed carbon nanotube electrodes with low sheet resistance for electrochemical sensor applications, J. Electrochem. Soc. 161 (2014) 83044-83048.

[48] T.H. da Costa, E. Song, R.P. Tortorich, A paper-based electrochemical sensor using inkjet-printed carbon nanotube electrodes, ECS J. Solid. State. SC 4 (2015) 53044-53047

[49] J. Zhao, Y. Yu, B. Weng, W. Zhang, A.T. Harris, A.I. Minett, Z. Yue, X.F. Huang, J. Chen, Sensitive and selective dopamine determination in human serum with inkjet printed Nafion/MWCNT chips, Electrochem. Commun. 37 (2013) 32-35.

[50] K. Kordas, T. Mustonen, G. Toth, H. Jantunen, M. Lajunen, C. Soldano, S. Talapatra, S. Kar, R. Vajtai, P.M. Ajayan, Inkjet printing of electrically conductive patterns of carbon nanotubes, Small 2 (2006) 1021-1025.

[51] J.P. Metters, C.E. Banks, in: D.W.M. Arrigan (Ed.), Carbon Nanomaterials in Electrochemical Detection, Electrochemical Strategies in Detection Science, Royal Society of Chemistry 2016, pp. 229-278.

[52] K. Scida, P.W. Stege, G. Haby, G.A. Messina, C.D. Garcia, Recent applications of carbon-based nanomaterials in analytical chemistry: critical review, Anal. Chim. Acta 691 (2011) 6-17.

[53] S.K. Vashist, D. Zheng, K. Al-Rubeaan, J.H.T. Luong, F.S. Sheu, Advances in carbon nanotube based electrochemical sensors for bioanalytical applications, Biotechnol. Adv. 29 (2011) 169-188.

[54] N. Yang, G.M. Swain, X. Jiang, Nanocarbon electrochemistry and electroanalysis: current status and future perspectives, Electroanalysis 28 (2016) 27-34. 
[55] A. Lesch, S. Maye, M. Jović, F. Gumy, P. Tacchini, H.H. Girault, Analytical sensing platforms with inkjet printed electrodes, Biotech, Biomaterials and Biomedical: TechConnect Briefs (2016) 121-124.

[56] R. Abbel, T. van Lammeren, R. Hendriks, J. Ploegmakers, E.J. Rubingh, E.R. Meinders, Photonic flash sintering of silver nanoparticle inks: a fast and convenient method for the preparation of highly conductive structures on foil, MRS Communications 2 (2012) 145-150.

[57] K.A. Schroder, S.C. McCool, W.F. Furlan, Broadcast photonic curing of metallic nanoparticle films, NSTI Nanotech 2006 Technical Proceedings, 3, 2006, pp. 198-201.
[58] G. Volpe, R. Draisci, G. Palleschi, D. Compagnone, 3,3',5,5'-Tetramethylbenzidine as electrochemical substrate for horseradish peroxidase based enzyme immunoassays. A comparative study, Analyst 123 (1998) 1303-1307.

[59] J.R. Garber, R.H. Cobin, H. Gharib, J.V. Hennessey, I. Klein, J.I. Mechanick, R. PessahPollack, P.A. Singer, K.A. Woeber, Clinical practice guidelines for hypothyroidism in adults: cosponsored by the American Association of Clinical Endocrinologists and the American Thyroid Association, Endocr. Pract. 18 (2012) 988-1028. 